\title{
Fußball zwischen Krieg und Frieden. Anmerkungen zu einem fast philosophischen Thema
}

\author{
Hans-Georg Ehrhart*
}

\begin{abstract}
According to the official FIFA jargon, soccer is supposed to be an instrument for a better understanding between people and a force for peace. It is also a force with the potential to electrify the masses. Therefore, politicians sometimes like to instrumentalize soccer for political ends including war. In this essay we approach the topic "Soccer between war and peace" from several angles: Civilisation and violence, identity and nation, soccer in dictatorships, soccer and war, war and peace, development and peace. We conclude that soccer is neither good nor bad. It is what we make of it. Soccer is not a substitute of war and revolutions. It can be misused and does not rule the world. However, soccer can also be a force for good.
\end{abstract}

Keywords: Soccer, war, peace, civilisation, development

Stichworte: Fußball, Krieg, Frieden, Zivilisierung, Entwicklung

\section{Einleitung}

$\mathrm{D}$ ie WM in Brasilien begann am 12. Juni 2014 um 22.00 mit dem Spiel Brasilien gegen Kroatien. Vor dem Startpfiff wurde eine symbolische Friedensinitiative bemüht. Drei Kinder ließen je eine Friedenstaube in die Lüfte steigen. Ausgedacht hatten sich diese Geste das Friedensnobelkomitee und der Weltfußballverband FIFA. Gleichzeitig gab es in verschiedenen Städten des Landes gewaltsame Auseinandersetzungen zwischen der Polizei und Kritikern dieser Großveranstaltung, die der eigenen Regierung milliardenschwere Geldverschwendung und der FIFA Neokolonialismus vorwarfen.

Das hat natürlich nichts mit Krieg zu tun, allenfalls mit partiell gewaltsamen sozialen Konflikten. Allein dieser Vorfall zeigt: Fußball ist nicht per se unpolitisch, wie hin und wieder behauptet wird. ${ }^{1}$ Es handelt sich immerhin um die weltweit beliebteste Sportart, die Zugang zu einem Millionenpublikum ermöglicht. Fußball gehört heute wohl zu den am stärksten globalisierten Sportarten. Seine Faszination wirkt in unterschiedlichen kulturellen Kontexten. Sie lässt sich politisch nutzen, im positiven und im negativen Sinne. Bereits der Aufmarsch von Staats- und Regierungschefs, von Ministern und anderen Politikern bei Großveranstaltungen vermittelt einen ersten Eindruck davon, dass Fußball von politischer Relevanz sein kann. Die FAZ schrieb angesichts der in ausländischen Medien kritisch kommentierten Tatsache, dass der Prozess gegen einen Fußballmanager - gemeint war Uli Hoeneß vom FC Bayern München - die Krimkrise aus den Schlagzeilen verdrängt habe, zutreffend: „Der Fußball ist eine Macht im Staat“. Er habe positive Auswirkungen, aber auch eine manipulative Kraft, deren sich Machtmenschen gerne bedienten. ${ }^{2}$

* Dr. Hans Georg Ehrhart ist Leiter des Zentrums für EUropäische Friedensund Sicherheitsstudien (ZEUS) am Institut für Friedensforschung und Sicherheitspolitik an der Universität Hamburg (IFSH).

Dieser Beitrag ist eine überarbeitete, erweiterte und aktualisierte Fassung von Hans-Georg Ehrhart, Fußball und Völkerverständigung, in: Aus Politik und Zeitgeschichte, 19/2006, unter: http://www.bpb.de/apuz/29765/fussballund-voelkerverstaendigung?p=all (Zugriff: 13.6.2014).

1 Bereits 1986 kritisierte Lincoln Allison (Sports and Politics, in: ders., The Politics of Sports, Manchester 1986, S. 17-21) den Mythos vom unpolitischen Charakter des Sports.

2 Anno Hecker, Von dieser Welt, in: Frankfurter Allgemeine Zeitung, 15.3.2014, S. 1.
Artikel 2 der Statuten der FIFA gibt als Zweck dieser internationalen Nichtregierungsorganisation an, den Fußball „weltweit zu verbreiten, wobei der völkerverbindende, erzieherische, kulturelle und humanitäre Stellenwert des Fußballs berücksichtigt werden soll“ ${ }^{3}{ }^{3}$ Der selbstherrliche und durch Korruptionsskandale belastete FIFA-Präsident Josef Blatter beschreibt die Rolle des Fußballs als die einer weltweiten Friedensorganisation, wenn er sagt: „Fußball vereinigt die Völker und kann mit seiner positiven Energie einen großen Beitrag in einer bösartigen und verrückten Welt leisten“. ${ }^{4}$

Kontrastiert wird dieses Bemühen leider allzu oft von den hässlichen Realitäten in dieser Welt. Vergleichsweise harmlos erscheint noch der bellizistische Sprachgebrauch, dessen sich insbesondere die Medien manchmal bedienen. So beschrieb der ZDF-Fernsehreporter Béla Réthy das Länderspiel Deutschland Frankreich am 12. November 2005 als "Abnutzungskampf“, um kurz darauf zu ergänzen, es sei ganz wichtig für die Moral, die Franzosen in Kleinkriegen zu bekämpfen. Nun kann man dem jovialen Reporter natürlich keine verbale Kriegstreiberei unterstellen, sondern eher einen unkritischen Sprachgebrauch - und da ist er gewiss kein Einzelfall. Im Fußballjargon wird ja seit jeher "gebombt", "der Gegner ausgeschaltet", der „Killerinstinkt" beschworen, „aus allen Rohren geschossen“, eine „wahre Abwehrschlacht geliefert" und „bis aufs Messer gekämpft“. Weitaus schlimmer sind Gewaltakte in und vor den Stadien, der Hooliganismus und die Funktionalisierung des Fußballs für das Schüren von gesellschaftlichen oder internationalen Konflikten.

Um einen Zugang zum Thema „Fußball zwischen Krieg und Frieden" zu erhalten, müssen also auch einige kritische Aspekte angesprochen werden - wenn auch gelegentlich mit einem Augenzwinkern. Das soll im Folgenden zu den Stichworten Zivilisierung der Gewalt, Identität und Nation, Fußball in Diktaturen, Fußball und Krieg sowie entwicklungs- und friedenspolitisches Potenzial des Fußballs versucht werden.

3 FIFA-Statuten, unter: http://www.fifa.com (Zugriff: 13.6.2014).

4 Josef Blatter auf der Münchener Konferenz „Visions of Football“, unter: www.bayern2006.de/home/events/visions_blatter_auftakt.htm (Zugriff: 23.8.2005) 


\section{Zivilisierung der Gewalt}

Das Martialische kann als eine Facette des kulturellen Phänomens Fußball auf eine lange Geschichte zurückblicken. Die Legende berichtet, dass Fußball auf einen kriegerischen Vorfall vor ca. tausend Jahren zurückgeht. Damals sollen die Engländer nach ihrem Sieg dem dänischen Aggressor den Kopf abgehackt und damit Fußball gespielt haben. Auch wenn dieser Geschichte kein Glauben geschenkt wird, so ist doch historisch belegt, dass Fußball - oder das, was man in früheren Zeiten auf der Insel darunter verstand - ein eher derbes Raufen war, das im 14. und 15. Jahrhundert mehrfach wegen seiner Brutalität verboten wurde. ${ }^{5}$

Im Laufe des 19. Jahrhunderts setzte ein Zivilisierungsprozess ein, der im wichtigsten Datum der modernen Fußballgeschichte mündete. Am 26. Oktober 1863 wurde in England die Football Association gegründet, die das weitgehend bis heute gültige Regelwerk für das Fußballspiel definierte und kodifizierte. ${ }^{6}$ Mit dieser Verregelung wurde ein entscheidender Schritt zur Einhegung der Gewalt getan. Seitdem gilt: Fußball ist ein auf Regeln basierendes Spiel und eben kein Krieg, wie es der holländische Trainer Rinus Michels einmal gesagt haben soll. Im Krieg geht es um die Vernichtung des Feindes, im Fußball um den sportlichen Wettkampf mit einem Gegner. Im Gegensatz zum Krieg ist die Grundstruktur des Fußballs kooperativ und egalitär. Ohne eine gegnerische Mannschaft wäre kein Spiel möglich. Gespielt wird nach für alle gültigen Regeln, die vom Schiedsrichter und gegebenenfalls von der zuständigen Sportgerichtsbarkeit durchgesetzt werden. Insofern ist die Fußballwelt der internationalen Politik und deren Fähigkeit zur friedlichen Konfliktregelung weit voraus.

Gleichwohl wird der Fußball immer wieder von Gewaltakten begleitet, seien es handgreifliche Auseinandersetzungen auf dem Platz, auf den Rängen oder außerhalb des Stadions. Erinnert sei nur an die Katastrophe, die sich Mitte der 1980er Jahre im Brüsseler Heysel-Stadion ereignete, als die Fans von Juventus Turin in panischer Furcht vor einem aus Anhängern des FC Liverpool bestehenden Mob sich an den Rand ihres Blocks flüchteten und dort grausam zu Tode gequetscht wurden. Oder an das Schicksal des französischen Polizisten David Nivel, der von deutschen rechtsradikalen Hooligans beinahe tot geprügelt wurde und seither schwerbehindert ist. Gehört Gewalt also zum Fußball? Meiner Einschätzung nach lautet die Antwort: nicht zwangsläufig. Der Fußball ist ein Spiegelbild der Gesellschaft und ihres Gewaltpotenzials. Wie ist die Gewalt zu erklären? Rührt sie etwa daher, dass es sich traditionell um einen „Männerkampfsport“ mit entsprechendem Imponiergehabe handelt? Die von mir geteilte Antwort von Klaus Theweleit lautet: „Ganz auszuschließen ist ein solcher Anteil am konkurrierenden Bolzgehabe in der Tat nicht" ${ }^{7}$ Fußball kann zweifellos militarisieren - glücklicherweise betrifft dies bislang nur eine kleine Minderheit -, er kann aber auch zivilisieren, indem er die in jedem steckenden Aggressionspotenziale kanalisiert. Ja, er kann auch zur Förderung sozialer Solidarität und Integration beitragen. Es hängt letztlich von der Bedeutung bzw. dem Sinn $\mathrm{ab}$, den wir dem Fußballspiel und der typischen Rivalität zwi-

5 Vgl. Paul Auster, Der perfekte Kriegsersatz, in: Zeitschrift für Kultur-Austausch, $1 / 2000$, S. 62.

6 Vgl. zur Frühgeschichte des Fußballs Allen Guttmann, Games and Empires. Modern Sports and Cultural Imperialism, New York 1994.

7 Klaus Theweleit, Tor zur Welt. Fußball als Realitätsmodell, Köln 2004, S. 94. schen zwei gegnerischen Mannschaften bei der Konstruktion unserer sozialen Wirklichkeit beimessen.

\section{Identität und Nation}

Fußball hat viel mit Identität zu tun. Man könnte mit einem der Begründer der konstruktivistischen IB-Theorie, Alexander Wendt, formulieren: „Football is what you make of it." ${ }^{8}$ Idee und Identifikation sind prägend. Man ist entweder Anhänger der einen oder der anderen Mannschaft. Gerade Mannschaftssportarten vermitteln einen starken Sinn für exklusives Gruppenverhalten. Es ist unstrittig, dass Fußballvereine kulturelle Identitäten durch Rivalität und Differenz prägen. Das wird immer dann besonders deutlich, wenn Derbys anstehen, so etwa wenn Celtic Glasgow gegen die Rangers spielt, AS Rom gegen Lazio oder der Hamburger SV gegen den FC St. Pauli. Das dyadische Drama wird nicht selten überlagert von geografischen, sozialen, politischen, religiösen oder ethnischen Selbstzuweisungen, die identitätsverstärkend wirken. Der hochkompetitive Charakter des Fußballs manifestiert sich auf internationaler Ebene durch nationale Antagonismen und Rivalitäten. Man denke nur an die Länderspiele zwischen Deutschland und den Niederlanden, Griechenland gegen die Türkei, Kroatien gegen Serbien oder Deutschland gegen England. Bei diesen Anlässen können historische Rivalitäten mit fußballerischen Mitteln „durchgespielt“, aber auch Stereotype und Ressentiments wiederbelebt oder verstärkt werden.

So wurde durch das brutale Foul des deutschen Torhüters Harald „Toni“ Schumacher gegen den französischen Spieler Patrick Battiston 1982 bei der WM in Spanien das alte Bild vom hässlichen Deutschen wiederbelebt. Erschwerend kam hinzu, dass Frankreich die eindeutig bessere Mannschaft stellte und Schumacher in arroganter Manier öffentlich anbot, dem Gefoulten die Jacketkronen zu bezahlen. Noch 23 Jahre später wurde Patrick Battiston, der zu diesem Zeitpunkt für die Nachwuchsförderung im französischen Fußball zuständig war, im Rahmen einer Podiumsveranstaltung auf dieses Foul und seine Auswirkungen angesprochen und er sagte: „Das Foul hat ziemlich böse ausgesehen und rief eine gewaltige Reaktion hervor. Mir war berichtet worden, dass seine [Schumachers] Familie, seine Kinder bedroht wurden und in der französischen Presse wurden plötzlich die Ereignisse von 1939 bis 1945 als Vergleich herangezogen. Das ging mir alles viel zu weit, und ich wollte die Eskalation nicht. Man weiß ja nie, was aus so etwas werden kann. ... Da habe ich gesagt: Schluss, aus. Lass uns die ganze Sache vergessen und nach vorne blicken".

Folgt man der These von Alan Tomlinson, dann finden Nationen ihren deutlichsten Ausdruck „einerseits in kriegerischen, andererseits in sportlichen Auseinandersetzungen $“{ }^{10}$ Fußball scheint besonders für die Herstellung von nationalen Gefühlen und Identität geeignet, weil sich auf dieser binären Folie das jeweilige

8 Frei nach Alexander Wendt: Anarchy is what states make of it: the social construction of power politics, in: International Organization 2/1992, S. 391-425.

9 Podiumsdiskussion mit Patrick Battiston beim Fußballclub Girondins de Bourdeaux am 28.07.2005.

10 Alan Tomlinson, FIFA and the World Cup: The Expanding Football Family, in: John Sugden, Alan Tomlinson (Hrsg.), Hosts and Champions. Soccer Cultures, National Identities and the USA World Cup, Aldershot 1994, S. 14 
nationale Konzept besonders gut repräsentieren lässt. Man denke nur an die Wirkung des deutschen WM-Sieges von 1954 auf das Selbstbewusstsein der Deutschen. ${ }^{11}$ Der brasilianische Schriftsteller Joao Ubaldo Ribeiro beschreibt den ersten brasilianischen Gewinn der Fußball-WM im Jahre 1958 als den eigentlichen nationalen Gründungsakt, 69 Jahre nach der Erlangung der politischen Unabhängigkeit. ${ }^{12}$ Ein weiteres Beispiel für nationale Repräsentationen durch Fußball ist der WM-Sieg Frankreichs 1998, der die größte Demonstration öffentlichen Glücksgefühls und denselben Ausbruch des Nationalstolzes bewirkt haben soll, wie nach der Befreiung von den Deutschen im Jahr $1944 .^{13}$

Obwohl Fußball vom Selbstverständnis her eigentlich universalistisch ist, spielen nationale Grenzen auch im Zeitalter der Globalisierung eine wichtige Rolle. Nach Artikel 10 des FIFA-Statuts können i.d.R. nur Verbände Mitglied werden, die in einem von der Staatengemeinschaft anerkannten Land für die Organisation und Kontrolle des Fußballs verantwortlich sind. Fußball und Staatlichkeit bilden also in gewissem Sinne eine Einheit. Fußball wird auch von Staaten politisch eingesetzt, um z.B. im Rahmen der auswärtigen Kulturpolitik ein positives Bild von sich zu zeichnen. Auch das kann abhängig von Inhalt, Kontext und Art der Kommunikation der Völkerverständigung dienen. Fußball kann sogar dabei helfen, geteilte Nationen zusammenzuführen, sei es kurzfristig ideell, wie 1954 bei den Deutschen aus Ost und West oder perspektivisch im Rahmen der Sportdiplomatie, wie bei den vorsichtigen Annäherungsversuchen zwischen Südund Nordkorea. Beide Staaten, die sich offiziell immer noch im Kriegszustand befinden, feierten im Sommer 2005 erstmals gemeinsam das Ende des Zweiten Weltkriegs. Auf dem Programm stand auch ein Fußballspiel, dass Südkorea vor mehr als 60.000 Zuschauern mit 3:0 gewann. ${ }^{14}$ Unter die Rubrik Sportdiplomatie fällt auch das Länderspiel Nordkorea gegen Japan vom Februar 2005. Immerhin fühlt sich Japan von der nordkoreanischen Nuklear- und Raketenrüstung bedroht. Gleichwohl machte der Fußball es möglich, dass diese Begegnung stattfand und mehrere Tausend (nord)koreanisch stämmige Japaner ihre Mannschaft unterstützen konnten. ${ }^{15}$ Gleichwohl: Grundsätzlich geändert hat sich das politische Verhältnis zwischen beiden Ländern nicht. Das Qualifikationsspiel beider Koreas für die WM 2010 musste in Shanghai stattfinden, weil Nordkoreas Kim Jong-il es unerträglich fand, sich in Pjöngjang die südkoreanische Nationalhymne anhören zu müssen. ${ }^{16}$

\section{Fußball und Diktatur}

Wir wissen nicht viel über die geschlossene Gesellschaft in der Diktatur Nordkorea, aber so viel ist bekannt: Die Nordkoreaner sind fußballbegeistert und nahmen als Überraschungsmannschaft 1966 an der WM in England teil. Welche Rolle spielt also

11 Vgl. Norbert Seitz, Was symbolisiert das „Wunder von Bern“?, in: Aus Politik und Zeitgeschichte, 26/2004, S. 3-6.

12 Joao Ubaldo Ribeiro, Aber der Ball gehört uns, in: Zeitschrift für KulturAustausch, 1/2000, S. 72-75

13 Paul Auster (Anm. 6), S. 62.

14 Vgl. www.stern.de/politik/ausland/index.html?id=544291\&nv=pr\&pr= (Zugriff: 2.11.2005)

15 Vgl. www.tokyo.diplo.de/de/02/Botschafter_und_Abteilungen/P... (Zugriff: 3.11.2005).

16 Frank Hollmann, Ein großer Moment - auch ohne Tor, in: Frankfurter Allgemeine Zeitung 27.3.2008, S. 35.
Fußball in einer unfreien Gesellschaft? Bereits in der Zwischenkriegszeit der ersten Hälfte des 20. Jahrhunderts wurde deutlich, dass das Verhältnis von Fußball und Diktatur sehr unterschiedlich sein kann und differenziert betrachtet werden muss. Wenn es die vorherrschende Ideologie und Politik wollte, wurde auf den Fußball und die Fußballer keine Rücksicht genommen. Die Schergen Stalins und Hitlers scheuten vor Willkürakten nicht zurück, wenn es um die Durchsetzung ihres politischen Willens ging. So starb Walter Bensemann, der Mitbegründer des DFB, Gründer des FC Bayern München - des sogenannten „Judenclubs“ - und der Fachzeitschrift „Kicker“ nach seiner Enteignung durch die Nazis mittellos in der Schweiz. Andere, wie der Rekordtorschütze Gottfried Fuchs, mussten emigrieren oder kamen, wie etwa Julius Hirsch, in Konzentrationslagern um. ${ }^{17}$ Für andere Diktaturen waren der Fußball und der mit seiner Hilfe zusätzlich befeuerte Nationalismus ein willkommenes Mittel, um die eigene Größe zu zelebrieren. So unterstützte das faschistische Regime Italiens die Entwicklung des nationalen Fußballs und stilisierte den nicht zuletzt durch „großzügiges Entgegenkommen“ der Schiedsrichter ermöglichten Gewinn der WM 1934 und 1938 zum Ausdruck nationaler Größe und ideologischer Überlegenheit.

Mag man den Fans zugute halten, dass sie vor lauter nationaler Begeisterung die politische Funktionalisierung des Fußballs nicht erkennen. So ist es umso wichtiger, dass Verbände und Politik von außerhalb Position beziehen. Ein gutes Beispiel dafür ist der Verband des Mutterlandes des Fußballs, die Football Association, die 1928 aus Protest gegen die zunehmende Politisierung dieses Sports durch Diktaturen aus der FIFA aus- und erst nach dem II. Weltkrieg wieder beitrat. ${ }^{18}$ In Deutschland brauchte der zeitweise konservativ bis reaktionär durchwirkte DFB über ein halbes Jahrhundert, ehe er sich 2001 dazu durchrang, seine Verstrickungen in das NS-System von einem Historiker untersuchen zu lassen. Rechtzeitig vor Beginn der WM in Deutschland legte Nils Havemann seine Studie „Fußball unterm Hakenkreuz" vor, in der er darlegt, wie vorbehaltlos sich der DFB in den Dienst des NS-Staates stellte. ${ }^{19}$

Noch 1978 war es zu einem Skandal bei der WM im diktatorisch regierten Argentinien gekommen, als der DFB jenen rechtsradikalen Kampfflieger Hans-Ulrich Rudel in das Mannschaftsquartier der deutschen Nationalelf einlud, dessen Teilnahme an einem Traditionstreffen in einer Bundeswehrkaserne zwei Jahre zuvor zur Entlassung der verantwortlichen Generäle durch Verteidigungsminister Georg Leber geführt hatte. Die fehlende politische Sensibilität verdeutlichte der damalige Spieler Herbert Zimmermann vom 1. FC Köln. Danach gefragt, ob er überhaupt schlafen könne, wenn in der Nähe Gefangene gefoltert würden, entgegnete er: „Wieso? Wir haben ja die GSG 9 bei uns.“ 20

Dass eine Fußballweltmeisterschaft angesichts der wachsenden Medienpräsenz nicht unpolitisch ist, war bereits 1974 in der Bundesrepublik Deutschland unter Beweis gestellt worden. Ein Jahr zuvor waren 5000 Flüchtlinge vor den chilenischen Put-

17 Vgl. Dietrich Schulze-Marmeling (Hrsg.), Davidstern und Lederball, Göttingen 2003.

18 Vgl. Christiane Eisenberg, Fußball als globales Phänomen. Historische Perspektiven, in: Aus Politik und Zeitgeschichte, 26/2004, S. 12.

19 Vgl. Nils Havemann, Fußball unterm Hakenkreuz. Der DFB zwischen Sport, Politik und Kommerz, Frankfurt/New York 2005.

20 Norbert Seitz, Von Bern bis Los Angeles, in: Aus Politik und Zeitgeschichte, $24 / 94$, S. 8 
schisten nach Deutschland geflohen, davon ein Drittel in die DDR. Sie organisierten sich in Chile-Komitees und machten mit Aktionen während des Spiels der chilenischen Mannschaft auf die Lage in ihrem Heimatland aufmerksam. Die Bilder wurden in die ganze Welt hinausgetragen und sollen sogar von Häftlingen in Chile gesehen worden sein. Diese Aktionen waren Teil eines politischen Kampfes mit Hilfe von "soft power", die auf weiche, kulturelle Faktoren wie Information und Bilder setzt.

Diktaturen neigen bekanntermaßen zu härteren Mitteln bei der Durchsetzung ihres Herrschaftsanspruchs. Der Fall Lutz Eigendorf hat gezeigt, dass sie auch vor Mord an einem bekannten Fußballer im Ausland nicht zurückschrecken. Eigendorf hatte sich 1979 nach einem Spiel des vom Ministerium für Staatssicherheit (MfS) geförderten Eliteclubs BFC Dynamo Berlin gegen den 1. FC Kaiserslautern abgesetzt und spielte in der Folge für den 1. FC Kaiserslautern und Eintracht Braunschweig. Ihm wurde zum Verhängnis, dass MfS-Chef Erich Mielke ein begeisterter Fußballanhänger war und Eigendorfs DDR-kritische Äußerungen in westdeutschen Medien als Feindpropaganda wertete, die er mit der Ermordung des Fußballers bestraft haben soll.

Während dieser Mord verdeckt geschah und erst nach der Öffnung der Stasi-Archive mit an Sicherheit grenzender Wahrscheinlichkeit aufgeklärt werden konnte, ${ }^{21}$ wurde im Irak des Saddam Hussein die Willkür, der auch Fußballer in Diktaturen ausgesetzt sein können, offen ausgeübt. Saddams Sohn Udai war 18 Jahre lang Vorsitzender des irakischen Fußballverbandes. Da er der Ansicht war, Fußballer könne man zum Erfolg zwingen, stand auf Niederlagen der Nationalmannschaft bis zu zwei Wochen Haft und gegebenenfalls die Peitschenstrafe. Seine Devise war offenbar: „Na ja, man muss den Burschen nur Beine machen“. Erreicht wurde mit dieser Brachialmethode das Gegenteil: Unter seiner Regentschaft wurde der einstmals erfolgreiche irakische Fußball durch Korruption, politische Gängelei und persönliche Willkür heruntergewirtschaftet. ${ }^{22}$

Wozu die politisch-religiöse Durchdringung des Fußballs führen kann zeigt ein Bericht aus der International Herald Tribune, der im Oktober 2005 unter der Überschrift „Shariah on the soccer field. God is in the rules" erschienen ist. Darin wird eine Fatwa, also eine islamische Rechtsposition, wiedergegeben, deren Umsetzung auf die Abschaffung des heutigen Fußballs hinauslaufen würde. Das Argument lautet, dass die internationalen Regeln von Häretikern gemacht worden seien und nicht den Regeln der Scharia entsprächen. Fußball solle kein vergnüglicher Zeitvertreib sein, sondern ausschließlich der körperlichen Ertüchtigung für den „heiligen Krieg“ (Dschihad) dienen. Fußball wird also immer wieder für die eigenen ideologischen Ziele instrumentalisiert. ${ }^{23}$

\section{Fußball und Krieg}

Dieser zivilisatorische Rückschritt - immerhin hat der regelbasierte moderne Sport nach Norbert Elias und Eric Dunning zur

21 Vgl. Herbert Schwan, Der lange Arm der Stasi: „Tod dem Verräter!“, München 2000 .

22 Vgl. Heike Faller, Zwischen Krieg und Spiel, in: Die Zeit, Nr. 36/2003, unter: http://zeus.zeit.de/text/2003/36/Irak_36 (Zugriff: 24.8.2005).

23 Geoff D. Porter, Sharia on the soccer field. God is in the rules, in: International Herald Tribune, 17.10.2005.
Zivilisierung und damit auch Pazifizierung der europäischen Gesellschaften beigetragen ${ }^{24}$ - war gut hundert Jahre früher durchaus auch im Europa des Imperialismus an der Tagesordnung. Übersteigerter Nationalismus und Großmachtphantasien ließen den Fußball zu einer Art militärischer Vorbereitung auf den nächsten Ernstfall verkommen. Das Bemühen der internationalen Friedensbewegung, mittels der olympischen Idee und internationaler Fußballbegegnungen Krieg zu verhindern, erwies sich gegenüber dieser aggressiven „Sinngebung“ als chancenlos. Während des Ersten Weltkrieges wurde hinter den Linien sogar ein regelrechter Trainingsbetrieb organisiert, um die Moral der Soldaten durch Fußball aufrechtzuerhalten.

Dass Fußball bei alledem etwas zutiefst Verbindendes über die Schützengräben hinweg behalten und gleichzeitig weit davon entfernt sein kann, verdeutlicht Christian Carion in seinem Film „Merry Christmas“. Die dem Film zugrunde liegende wahre Begebenheit ereignete sich Weihnachten 1914 an der Westfront. Deutsche, britische und französische Soldaten vereinbaren eine kurze Waffenruhe, während der sie sich außerhalb der Schützengräben im Niemandsland treffen, sich als menschliche Wesen erfahren und u.a. miteinander Fußball spielen. Aufgrund dieser nicht erwünschten „Verbrüderung mit dem Feind" werden die Einheiten aufgelöst oder an andere Frontabschnitte verlegt, um den Krieg wieder ganz im Sinne der staatlichen Hasspropaganda fortzusetzen. ${ }^{25}$

Ein Fußballspiel unter noch grausameren Bedingungen fand im August 1942 im besetzten Kiew statt. Die Betriebsmannschaft der Brotfabrik Nr. 3, bestückt mit ehemaligen Stars der Kiewer Fußballvereine Dynamo und Lokomotive, spielte gegen die Vorzeigemannschaft der deutschen Luftwaffe - und schlug diese vor Tausenden von Zuschauern mit 5:1. Sie hatten nicht nur ein Fußballspiel gewonnen, sondern den Besatzer deklassiert. Nachdem sie auch das Rückspiel gewonnen hatten, wurden sie wenig später abgeführt. Die These, dass sie wegen des Fußballspiels umkamen, ist unbewiesen, aber nicht die Tatsache, dass sie der deutschen Vernichtungsmaschinerie zum Opfer fielen. ${ }^{26}$ Die Sinngebung dieses Fußballspiels war für die Ukrainer die Hoffnung, dass der scheinbar übermächtige Gegner doch besiegbar ist. ${ }^{27}$

Wurde Fußball in den hier erwähnten zwei Fällen während des Krieges gespielt, sei es um die Kampf- oder die Arbeitsmoral zu stärken, so diente er in einem Fall auch als Anlass für einen Krieg. Der 1969 ausgebrochene Krieg zwischen El Salvador und Honduras ging fälschlicherweise als „Fußballkrieg“ in die Geschichte ein. Was war geschehen? Während der Qualifikationsspiele zur WM kam es zu Krawallen, zunächst in Tegucigalpa, dann in verstärktem Maße während des Rückspiels in San Salvador, wo die Mannschaft aus Honduras in einem gepanzerten Fahrzeug ins Stadion gebracht wurde. Nachdem El Salvador das Entscheidungsspiel in Mexiko mit 3:2 für sich entschieden hatte, kam es zu Ausschreitungen gegen salvadorianische Immigranten in Honduras, die El Salvador mit dem Argument, die Menschenrechte der salvadorianischen Siedler zu schützen - Wladimir

24 Norbert Elias/Eric Dunning, Quest for Excitement. Sport and Leisure in the Civilizing Process, Oxford 1986.

25 Vgl. dazu Michael Jürgs, Der kleine Frieden im großen Krieg, München 2003.

26 Vgl. Erik Eggers, Das Todesspiel, in: Elf Freunde, März 2003, S. 26-27.

27 Vgl. zur Rolle des Fußballs zur Stärkung der nationalen Widerstandsmoral in Großbritannien Jack Rollin, Soccer at War, London 2005 
Putin lässt grüßen -, zur militärischen Intervention veranlasste. Der Krieg konnte zwar unter Vermittlung der Organisation für Amerikanische Einheit nach wenigen Tagen beendet werden, kostete aber über 2000 Menschen das Leben, ca. 6000 wurden verwundet, der Handel zwischen beiden Staaten brach zusammen, Zehntausende Menschen wurde vertrieben, das Projekt einer regionalen Wirtschaftsintegration Mittelamerikas wurde für lange Zeit auf Eis gelegt. Die eigentlichen Kriegsursachen waren jedoch nicht die Fußballspiele und die damit verbundenen Vorkommnisse. Es waren vielmehr wirtschaftliche und soziale Probleme, die Politiker auf beiden Seiten dazu veranlassten, aus machtpolitischem Kalkül mit Unterstützung der Medien auf die nationalistische Karte $\mathrm{zu}$ setzen. ${ }^{28}$

Folgt man der kruden Logik des serbischen Nationalisten Željko Ražnatović, der als Vorsitzender von Partizan Belgrad und Führer einer gefürchteten paramilitärischen Einheit im Jugoslawienkrieg zweifelhafte Berühmtheit erlangte, waren es die Auseinandersetzungen während des Fußballspiels zwischen Dynamo Zagreb und Roter Stern Belgrad am 13. März 1990, die bei ihm die Überzeugung reifen ließen, dass sich die Serben gegen die „kroatische Bedrohung“ zur Wehr setzen müssten. Natürlich war auch hier der Fußball nicht für den ein Jahr später ausbrechenden Krieg verantwortlich. Gleichwohl wurden Fußballereignisse als Bühnen benutzt, um den zunehmend aggressiven Nationalismus und das Ende des Vielvölkerstaates Jugoslawien zur Schau zu stellen und nationale Antagonismen zu verstärken. Die internationale Gemeinschaft bestrafte Jugoslawien wiederum damit, dass es von der Europameisterschaft 1992 ausgeschlossen wurde. Es war einer der ersten - eher symbolischen und letztlich erfolglosen - Schritte, um das Regime von Slobodan Milošević zum Einlenken zu bewegen. ${ }^{29}$

\section{Krieg und Frieden - fußballerische Analogien und pseudo-philosophische Anmerkungen}

Fußball hat eigentlich nur in symbolischer und semantischer Hinsicht etwas mit dem großen Thema Krieg und Frieden zu tun. Der britische Verhaltensforscher Desmond Morris hat das Fußballspiel als „rituelle Jagd, stilisierter Kampf und symbolisches Geschehen“ charakterisiert. ${ }^{30}$ Jagd und Kampf sind auch Wesensmerkmale von Krieg. Krieg wird manchmal verkürzt definiert als ein Kampf der Willen. Differenzierter ist der Zugang des deutschen Kriegsphilosophen Carl von Clausewitz, der das Wesen des Krieges mit der wundersamen Dreifaltigkeit zu erfassen versuchte. Diese besteht aus den in Wechselwirkung zueinander stehenden Wirkkräften Gewalt, Friktion und politische Zweckorientierung. Diesen ordnete er das Volk, die Armee und die Regierung zu.

Ähnlich wirkt auch im Fußball eine wundersame Dreifaltigkeit. Die erste Komponente ist der - allerdings friedliche - Zweikampf. Grenzüberschreitungen im Sinne des englischen Nationalspielers Gary Lineker kommen vor: „Abwehrspieler müssen vor allem

28 Vgl. Kennedy Hickman, Latin America: The Football War, unter: http:// militaryhistory.about.com/od/battleswars1900s/p/footballwar.htm (Zugriff: 14.6.2014).

29 Vgl. Pascal Boniface, Football as a Factor (and Reflection) of International Politics, in: The International Spectator, 4/1998, S. 87-98.

30 Zitiert in: http://zitate.woxikon.de/fussball (Zugriff: 10.6.2014). eines beherrschen: Mit einem herzlichsten Lächeln Stürmern in die Knochen treten. "31 Doch werden diese Fouls i.d.R. als solche erkannt und bestraft. Normeinhaltung und Abschreckung funktionieren also weitaus besser als im angebrochenen Zeitalter des postmodernen Krieges. Das zweite Element besteht auch im Fußball aus Friktionen und Zufall. Im Spiel interagieren zwei Mannschaften bzw. 22 Individuen und das Schiedsrichtergespann. Alle werden von außen beeinflusst. Das Spiel ermöglicht unzählige Handlungsoptionen, die nicht berechenbar sind. Um mit Jean-Paul Sartre zu sprechen: „Bei einem Fußballspiel verkompliziert sich allerdings alles durch die Anwesenheit der gegnerischen Mannschaft." 32 Sepp Herberger schrieb dieser Ungewissheit eine große Anziehungskraft zu: „Die Leute gehen ins Stadion, weil sie nicht wissen, wie es ausgeht. “33

Die dritte Komponente der fußballerischen Dreifaltigkeit ist der eigentliche Zweck des Spiels: der Sieg bzw. die Strategie, die zu ihm führt. Eine ganz eigene Dreifaltigkeit hatte Franz Beckenbauer, der Kaiser, im Sinn, als er sagte: „Ja gut, es gibt nur eine Möglichkeit: Sieg, Unentschieden oder Niederlage!“ - was philosophisch tiefsinnig darauf verweist, dass Strategien - wie im Krieg und im zivilen Leben - fehlschlagen können. Darum hatte Helmuth von Moltke (der Ältere) Strategie als „System von Aushilfen“ definiert, während sein Nachfolger Alfred Graf von Schlieffen bestrebt war, „Feldzüge mit der Vorhersehbarkeit und Genauigkeit preußischer Eisenbahnfahrpläne zu führen. “34 Wie im Krieg muss auch im Fußballspiel nicht nur die richtige Strategie ausgewählt werden. Ihre Umsetzung erfordert auch viel taktisches Geschick. So wie der Krieg nichts anderes als die Summe aller Gefechte ist, besteht ein Fußballspiel theoretisch aus der Summe aller Zweikämpfe, wobei ein guerillaähnliches taktisches Foul aus dem Hinterhalt entscheidend für Sieg oder Niederlage sein kann, aber nicht sein muss.

\section{Entwicklungs- und friedenspolitisches Potenzial}

Doch genug vom Krieg. Die fußballerische Dreifaltigkeit von Kampf, Zufall und Sieg kann friedenspolitisch gewendet werden in Spiel, Spontaneität und Spaß. Wenden wir uns also der positiven Rolle des Fußballs zu: seinem entwicklungs- und friedenspolitischem Potenzial. Kurz nachdem Präsident George W. Bush den Irakkieg offiziell für beendet erklärt hatte, kündigte die FIFA an, den irakischen Fußballverband mit 400.000 US-Dollar für den Aufbau seines zerstörten Hauptquartiers zu unterstützen. Australien, einer der engsten Alliierten der USA im Irakkrieg, lud die irakische Nationalmannschaft zu einem Match auf den fünften Kontinent ein. Das Spiel stand unter dem Motto „World Peace Game“. Bernd Stange, der damalige deutsche Cheftrainer der irakischen Mannschaft, lag wohl nicht ganz falsch, wenn er von Propagandaspielen sprach. ${ }^{35} \mathrm{Ihm}$ wäre es lieber gewesen, wenn die Amerikaner das Stadion, das sie als Militärbasis nutzten, geräumt und in einem geordneten Zustand übergeben hätten. Gleichwohl: Der Fußball sollte einmal mehr als Mittel zur Völ-

\footnotetext{
31 Ebenda.

32 Ebenda.

33 Ebenda.

34 Herfried Münkler: Der Große Krieg. Die Welt 1914-1918, Berlin 2013, S. 16 .

35 Vgl. Heike Faller (Anm. 22)
} 
kerverständigung dienen. Bereits am 13. August 2003 bestritt der Irak sein erstes offizielles Länderspiel nach dem Krieg. Gegner war Iran, der sportliche Erzrivale und Kriegsgegner von einst. Stanges Mannschaft siegte überraschend mit 1:0. Angesichts der katastrophalen Bedingungen zu Hause war es eine furiose Rückkehr des Irak auf die Bühne des internationalen Fußballs und ein erster Annäherungsschritt zwischen beiden Ländern. Deren Beziehungen sind inzwischen gut, der Irak befindet sich aber mittlerweile in einem brutalen Bürgerkrieg, in dem Fußball keinen Platz mehr hat.

Grundsätzlicher wird das Thema Völkerverständigung durch Sport und damit auch durch Fußball von den Vereinten Nationen angegangen. Seit Ende der 1990er Jahre entwickelte sich in diesem Rahmen ein zunehmendes Verständnis für den Zusammenhang von Entwicklung, Frieden und Sport. Ein erster Ausdruck davon war die Einrichtung einer internationalen Arbeitsgruppe und eines Büros mit der Bezeichnung „Sport für Entwicklung und Frieden“. ${ }^{36}$ Ende 2003 verabschiedete die Generalversammlung der Vereinten Nationen (VN) eine Resolution über Sport als Mittel zur Förderung der Bildung, der Gesundheit, der Entwicklung und des Friedens. ${ }^{37}$ In dieser Resolution werden die Regierungen $u$. a. gebeten, Sport als Instrument zu verwenden, das zur Verwirklichung der international vereinbarten Entwicklungsziele sowie der breiteren Ziele der Entwicklung und des Friedens beiträgt.

Der Sonderbeauftragte für Sport im Dienste von Entwicklung und Frieden des Generalsekretärs der Vereinten Nationen, der ehemalige Manager und Aufsichtsratsvorsitzende von Werder Bremen Willi Lemke, weist zu Recht darauf hin, dass der Sport und damit auch der Fußball - „über ein positives, kraftvolles Potential für die Friedensarbeit (verfügt), das es zu nutzen gilt. Sport dient als universelle Sprache, die verbindend wirkt und über politische, kulturelle und soziale Grenzen hinaus verstanden werden kann. Sport vermag es darüber hinaus, soziale Brücken zu bauen, wo andere Kommunikationsmittel oftmals versagen oder nicht existent sind. “38 Einige Beispiele illustrieren das:

So unterstützt das Entwicklungsprogramm der Vereinten Nationen (UNDP) zusammen mit der Weltbank, UNICEF und der GIZ seit 2010 in Kolumbien das Netzwerk Fußball und Frieden. Sein Ziel ist es, mit Hilfe von Fußball Frieden und Versöhnung unter Kindern, Jugendlichen und jungen Erwachsenen in diesem von Konflikten geplagten Land zu fördern. ${ }^{39}$ Die von Florian Zech gegründete Initiative AMANDLA EduFootball erhielt 2012 einen Preis für das beste Projekt in der Kategorie „Sport for Conflict Resolution“. Er belohnte den Ansatz der "Sport Safe Hubs“, die z.B. Sport und Bildungsaktivitäten in sozialen hot spots veranstalteten, etwa die Durchführung einer Nacht-Fußballliga Freitags- und Samstagsabends, um so die Kids und jungen Männer von Kriminalität und Drogenkonsum abzuhalten und ihnen

36 Vgl. z.B. Sport for Development and Peace, Report, 24. Mai 2005, www. un.org (Zugriff : 14.6.2014).

37 Vgl. A/RES/58/5 vom 17. November 2003, unter http://www.un.org/depts/ german/gv-58/band1/ar58005.pdf (Zugriff: 14.6.2014).

38 Willi Lemke zu Sport und Frieden am 10. Oktober 2012 auf dem Evangelischen Forum Annahof, unter: http://annahof-evangelisch.de/willi-lemkein-augsburg-sport-und-frieden/ (Zugriff: 10.6.2014).

39 UNDO, Playing soccer for peace in Columbia, http://www.undp.org/content/ undp/en/home/ourwork/povertyreduction/successstories/playing_soccer_forpeaceincolombia/ (10.6.2014). positive Erfahrungen zu vermitteln. ${ }^{40}$ Fußballprojekte werden also auch gestartet, um gewaltsame Konflikte zu überwinden und Versöhnungsprozesse zu verstärken. Der polyglotte Fußballtrainer Rudi Gutendorf berichtet von seiner Tätigkeit in Ruanda einige Jahre nach dem Völkermord, der fast eine Million Menschen das Leben gekostet hat: „Die Söhne habe ich dann vereint in der Nationalmannschaft. Und dann haben wir das Glück gehabt, Kenia im Afrika-Pokal zu schlagen. Da hat der Tutsi geflankt, und der Hutu eingeköpft. Da haben die vor Freude gekuschelt und sich geküsst, das ganze Stadion und das ganze Land. Seit der Zeit weiß ich, was Fußball bewirken kann“. ${ }^{41}$

Projekt FURD (Football Unites, Racism Divides) mit Sitz in Sheffield engagiert sich auf lokaler, nationaler und internationaler Ebene gegen Rassismus und für soziale Inklusion. ${ }^{42}$ In Hamburg fand Ende Mai 2014 zum 8. Mal das Antirarassistische Einladungsturnier statt, veranstaltet u.a. vom Fanprojekt des FC St. Pauli. Neben Fußball gab es Workshops und Diskussionsveranstaltungen zu politischen Themen. 35 Fangruppierungen aus 18 Ländern, die sich in ihren Stadien aktiv gegen Rassismus und Diskriminierung engagieren, sprachen über europaweite Vernetzung, die Rolle von Fans in sozialen Protestbewegungen und europäische Flüchtlingspolitik. Als Zeichen der Solidarität fand auch ein Fußballspiel zwischen dem FC Lampedusa und dem Antira-All Star Team im Millerntor-Stadion statt. ${ }^{43}$

Im Mittelpunkt vieler Basisprojekte steht der Straßenfußball. Dieser hat sich mittlerweile unter dem Namen „Streetfootballworld“ als eine globale Bewegung etabliert. Das Projekt hat eine eigene Organisation, gespielt wird nach eigenen Regeln. Über einen Sieg entscheiden nicht nur die Tore, sondern auch Fairplay und Genderaspekte. Es wird ohne Schiedsrichter gespielt. Das Projekt will Teamgeist, globales Lernen und ein Leben ohne Gewalt fördern. Fußball soll national und transnational als kultureller Mittler und Medium zur Förderung sozialer Entwicklung genutzt werden. ${ }^{4}$

\section{Schlussfolgerung}

Bill Shankley, der legendäre Manager des FC Liverpool hat einmal gesagt, Fußball sei keine Sache von Leben und Tod, sondern weitaus ernster. Dabei handelt es sich natürlich um eine mit britischem Humor konnotierte, nicht ganz ernst gemeinte Übertreibung. Fußball ist weder Krieg noch ein Allheilmittel für soziale oder politische Konflikte. Er ist von Natur aus weder gut noch böse. Er ist, was wir aus ihm machen. Er hat die Bedeutung, die wir ihm geben. Paul Auster ist leider nur tendenziell zuzustimmen, wenn er konstatiert: „Krieg und Revolutionen sind im Zeitalter der Medienherrschaft durch Fußball ersetzt worden“ ${ }^{45}$ Er beschreibt Fußball als den perfekten Kriegsersatz zumindest in Europa. Es gebe zwar randalierende Fans, aber man könne die Opfer an den Fingern zweier Hände abzählen. Eine Generation früher hätten wir in Millionen gezählt. ${ }^{46}$

40 Vgl. http://www.edufootball.org (Zugriff: 13.6.2014).

41 Interview mit Rudi Gutendorf, in: Elf Freunde, März 2003, S. 25

42 Vgl. http://www.furd.org/resources/Unity\%20Newsletter\%202013.pdf (Zugriff: 10.6.2014).

43 Vgl. Hamburger Abendblatt, 30. Mai 2014, S. 25.

44 Vgl. http://streetfootballworld.org (Zugriff: 12.6.2014).

45 Zit. in: Oliver Samson/Phillip Köster, In Ballgewittern, in: Elf Freunde, März 2003, S. 19.

46 Paul Auster, a.a.O. (Anm. 6) , S. 62. 
Der Bürgerkrieg in Syrien und der gewaltsamen Konflikt in der Ukraine relativieren diese positive Einschätzung. Dort, wo die Politik in falsche Bahnen gerät, ist auch der Fußball machtlos. König Fußball kann politisch missbraucht werden und regiert auf keinen Fall die Welt. Dennoch: Fußball kann weltweit gewissermaßen als Antibiotikum dienen, das bei bestimmten Symptomen zur Linderung oder Heilung von sozialen Problemen beitragen kann. Er hat zweifellos auf viele Menschen eine fast magische Wirkung. Doch was ist sein Geheimnis? Die
Antwort gibt kein geringerer als Uwe Seeler, der konstatierte: „Das Geheimnis des Fußballs ist ja der Ball.“47 Vom Ball wissen wir seit Altbundestrainer Sepp Herberger, dass er rund ist. Und wo muss das Runde gemäß Helmut Schulte hin? Genau, ins Eckige! Nach dieser grundlegenden, fast philosophischen Erkenntnis beende ich diesen Aufsatz mit den Worten von Giovanni Trappatoni: „Ich habe fertig!“

47 Zitiert in: http://zitate.woxikon.de/fussball (Zugriff: 10.6.2014).

\title{
Economic and Political Restraints for Reducing the Drug Economy in Afghanistan
}

Akmal Sokhibov*

\begin{abstract}
The stability of present-day Afghanistan is challenged by the pullout of international troops as well as the uncertainty of foreign aid receipt after 2014. But these problems will be mostly cushioned by the country's drug economy, which has influenced Afghan politics. It is questionable whether or not a new president of Afghanistan is able to survive politically if he attempts to eliminate the drug economy without offering better incentives for those involved. This article examines the role of the economy, foreign aid and political restraints for reducing the drug economy in Afghanistan. Furthermore, the article looks at alternative developments to the drug economy and the regional approach of Comprehensive Development.
\end{abstract}

Keywords: (Drug) economy, foreign aid, counter narcotics, alternative and comprehensive development

Stichworte: (Drogen) Ökonomie, Entwicklungshilfe, Drogenbekämpfung, alternative und vernetzte Entwicklung

\section{The Afghan economy after the collapse of the Taliban regime}

$\mathrm{D}$ espite long-term financial support and external aid, Afghanistan is still regarded as one of the poorest countries. The industrial sector of the economy has been developing slowly. Most companies and factories constructed by the Soviets in the 1960s and 1970s have either been destroyed or are not competitive. Traditional branches such as agriculture, trade and craftsmanship cannot make up for the current deficit (DAN 2012: 2). The government of Afghanistan is therefore working towards WTO membership with a modified and less complicated tax system, simplified licencing procedures as well as registration for companies and an improved export of goods. Taxes on imports were low and this resulted in the import of cheap goods, which also meant that goods and products made in Afghanistan could not compete on the market (DAN 2012: 2). But these state measures had a negative impact on local production. Due to the low tax and customs dues, Afghan goods and products were replaced by cheap imports and external goods. For example, a company in Herat that was responsible of producing tomato paste had to face bankruptcy due to low production costs in Iran and the low prices offered for the same product by Iran. Another good example is the printing business in Afghanistan, which could not develop, as large printing orders went to Pakistani (printing) companies due to low production and material costs, low customs charges for imports, and low transportation costs (DAN 2012: 18).
But the main issues for the economic development in Afghanistan are geographical conditions. The mountains that are dominating the landscape of Afghanistan are a hindrance to transport and infrastructure, and the dry climate is a challenge to the development of agriculture. Therefore, Afghanistan remains dependent on neighbouring countries such as Pakistan (see GIZ Afghanistan). In the years 2006-2010 the bilateral trade between Afghanistan and Pakistan increased from 850 million USD to $2.5 \mathrm{bn}$. USD. In 2010 and 2011 exports from Pakistan to Afghanistan amounted to $2.3 \mathrm{bn}$. USD. In contrast, within the same period of time Pakistan imported goods from Afghanistan only amounted to an official 172 million USD (Central Asia Online 01/20/2012). Mostly because of cheap goods from Pakistan, Afghan products have not been competitive within the region, and foreign aid has only to some extent been able to cushion the economic difficulties since 2002 .

\subsection{Foreign aid as a maintainer of the Afghan economy}

The contribution of foreign aid was decisive to the Afghan economy. The total expenditure for foreign aid from 2002 amounts to $62 \mathrm{bn}$. USD. The security sector has been the largest recipient of assistance. About $50 \%$ of foreign aid was given to the Afghan National Army and the Afghan National

\footnotetext{
PhD student at the Institute for Political Science of the University of Magdeburg in Germany. Master of Arts in Peace and Conflict Studies. This article has been double-blind peer-reviewed.
} 